\title{
An Overview of Risk Management
}

\author{
by Douglas G. Olson * and John A. Simkiss, Jr. **
}

\section{Introduction}

Risk and insurance management is merely the application of general concepts in scientific management to a particular problem. Like other areas of management, it is concerned with establishing or identifying objectives, gathering relevant information regarding the nature of the problem and the environment, evaluating the costs and benefits of alternatives using modern analytical techniques, and choosing the alternative that is most consistent with the goals and objectives of the enterprise. However, the complexity of the problem and alternative solutions have resulted in the indentification of risk management as being among the specialized management disciplines.

This discussion is devoted to a review of fundamental concepts that must be recognized in order to adequately understand the risk management problem. In specific, it has three goals :

(i) to analyze the objectives of risk management ;

(ii) to review the basic nature of the risk management problem; and,

(iii) to outline and analyze appropriate procedures.

Although risk management principles and procedures are applicable to a wide variety of problems, the examples and focus of this discussion will be limited. Concern will be directed to exposures to loss from such events as the destruction of property, legal liabilities to third parties, and occupational hazards to employees.

\section{The objectives of risk management}

A large part of risk management is essentially a specialized aspect of financial management. It has traditionally been distinguished from other financial management disciplines because of the unusual nature of the problem (vis-a-vis other financial management issues) and the solutions. The nature of the problem seems to have given rise to an equally unusual statement of objectives and the complexity of the solutions has necessitated an extensive body of knowledge related to complicated techniques for transferring the problem to some third party (such as an insurance company) and other solutions.

* Associate Professor at the Wharton School, University of Pennsylvania; Senior Associate, Insurance Research Services, Ltd. 07 Philadelphia.

** President of the Simkiss Companies. 
As students are often told in their introduction to the discipline, the concept of risk management has traditionally been associated with events that can only depress the overall economic well-being of the enterprise, widely referred to as pure risk events. Recognizing the dismal nature of the problem, equally dismal objectives have been devised. These have included statements to the effect that the goal should be to "minimize costs" or "minimize financial disruption." In fact, these objectives are no more appropriate to risk management than to any other financial management discipline. A parallel set of objectives in portfolio management would be the "maximization of returns" or "minimization of volatility in the value of the portfolio." The objectives are widely recognized as contradictory and neither is consistent with the composition of the overwhelming majority of investment portfolios. Financial theory suggests that a portfolio that minimizes volatility would be concentrating in short-term securities guaranteed by a financially solvent and stable government. On the other hand, maximization of the rates of return may well lead to a portfolio concentrating in commodities. In fact, the majority of investment portfolios strike a balance between the two extremes.

Virtually every enterprise faces the possibility of events of the pure risk variety. They are inherent in the very existence and nature of the enterprise. The strategy that is adopted for dealing with these risks will ultimately give rise to either greater or lesser costs than those that would have resulted from alternative strategies. Hence, they will directly affect profits. For example, if the enterprise decides to absorb the costs of occupationally-related injuries to workers using its own financial resources (i.e., decides not to purchase insurance), and no workers suffer injury, profits will be increased by the savings in premium that it would have otherwise expended for insurance. On the other hand, if the costs resulting from injuries to workers exceed the premium that would have been required, the profits of the enterprise will be lower than would have been the case if insurance had been purchased. Therefore, while the basic problem is of pure risk variety, the strategies are speculative in nature. The fact that risk management strategies affect profits by influencing costs rather than revenues has no substantive implication for the overall objectives.

With this in mind, it becomes apparent that the objective of the risk manager is the same as the objective of the portfolio manager or chief financial officer. It is to increase the wealth of shareholders or owners by selecting strategies that entail the optimal combination of expected returns and risk. The decision about whether to minimize financial disruption through the purchase of insurance, or, conversely, to internally assume the exposure to loss should be made on the same basis as any financial decision such as the investment in plant or equipment, whether to buy or lease, the choice of securities in an investment portfolio, and so forth.

\section{An overview of the problem}

As in other areas of the so-called management sciences, the last few years have witnessed a significant increase in the level of sophistication that has been applied to the risk management problem. Most of this sophistication has been attributable to the adoption of scientific problem solving techniques. Much more interest has been shown 
in scientific techniques for gathering and analyzing data and in the application of decision-making methodology based on modern theories of probability, statistics, and the measurement of risk. However, scientific methodology is not easily adaptable to many of the aspects of the problem, and when the process of risk management is viewed in its entirety, it remains as much an art as a science. Identification of the optimal strategy is still heavily dependent upon the intuition and imagination of individuals.

Like the portfolio manager, in evaluating alternatives the risk manager is concerned with two problems. The first is to define the ranges of possible outcomes under the different strategies. The fixed premium insurance strategy is straightforward: barring problems involving the financial integrity of the insurance company, there is no range. The outcome is a known cost equal to the premium. However, defining the range of outcomes under a pure self insurance strategy is complicated. While the maximum potential gain is known (equal to the savings in insurance premiums), the range of possible losses is subject to speculation. The problem is analogous to the age-old question of "how much liability insurance is enough?"

Although necessary, defining the ranges of possible outcomes is not sufficient. In order to evaluate the possibility that a strategy will result in financial disruption it is necessary to assess the likelihood that each of the possibilities in the range will actually occur. This is the essence of the concept of risk. On the one hand, the range of possible outcomes may be narrow and the expected result is overwhelmingly likely to actually occur. On the other hand, the range of possible outcomes could be extremely wide and the expected result no more likely to actually occur that any other possible outcome. Without this information it is virtually impossible to make appropriate decisions among alternatives.

Recognizing that risk is inversely related to the ability to predict introduces one of the most fundamental aspects of the risk management process. The ability to predict is critically dependent on both the quantity and reliability of information pertaining to the phenomenon being investigated. For some phenomena, large volumes of reliable and relevant information exist. In other cases, however, the uniqueness of the situation is such that available information is either nonexistent or the information that is available is so tangential that it is of little assistance. In this case, the extent of the risk that is involved is difficult to measure and hence difficult to evaluate. It is difficult under such circumstances to estimate the average expected costs, much less the likelihood associated with different outcomes in the range of possible results and hence the variations that might arise from one period to the next. In extreme cases, the very existence of the risk may be unknown.

The information problem becomes most apparent when the dynamic nature of the subject matter is fully recognized. The basic nature of the pure risk problem facing an enterprise is seldom static. On the one hand, changes in the nature of the enterprise may alter its exposure to loss. In addition, social, political, economic and other environmental changes usually exert some influence over the frequency with which pure risk events occur and their financial impact. Therefore, available information may reflect conditions that will not exist in the future and will have to be adjusted 
for anticipated changes in these underlying factors before it can be used as the basis for decision-making. While there are a variety of techniques for adjusting information from previous periods to reflect current conditions, anticipating future changes is a notoriously difficult problem.

In any event, since the ability to assess risk is a fundamental consideration in the choice among alternative strategies, the gathering and evaluation of basic information is a substantial part of the risk management process. For enterprises that are actively involved in risk management, maintaining adequate loss records reflecting their own experience and obtaining additional information from other sources is a critical part of the program.

This discussion turns attention to the basic steps that are involved in the risk management process and to an identification of alternatives. However, it may be possible to both clarify the risk management problem and objectives and to introduce the ensuing topics by drawing one further analogy. As is so often the case in financial management, the basic issues in risk management are twofold : the ability to predict and the ability to control. These two issues are aimed at the two basic types of choices involved in risk management : the selection of the appropriate financing mechanism and decisions regarding programs that are designed to mitigate the frequency or severity of pure risk events.

When the timing of events and their costs are easily predictable, they begin to resemble normal costs of doing business readily subjected to the budgeting process. Therefore, the question of which financing technique to use is much easier to answer in an objective, straightforward manner. However, the fact that costs are relatively predictable does not necessarily eliminate concern for programs that are designed to reduce these costs. These programs may still be desirable as long as their own costs are more than offset by the reduction in the potential frequency or severity of losses.

When the costs of the pure risk events are not easily predictable and the loss potential is significant, even greater attention is focused on the ability to control. It is often possible to devise programs that will not only reduce the frequency and severity of these events, but will also stabilize the costs and make them much more predictable. The epitome of the risk management problem arises when there are no programs available to control the occurrence of highly unpredictable financially disruptive events.

\section{The procedures of risk and insurance management}

Although there are significant differences among theoreticians and practitioners regarding the techniques that should be used, almost all agree on the fundamental procedures that must be followed. In their chronological order, these procedures include :

(i) identification of the pure risk events that may impose financial losses on the enterprise ; 
(ii) evaluation of the potential costs that would be involved if such events should occur ;

(iii) estimation of the frequency with which such events might occur ;

(iv) analysis of the alternatives that are available;

(v) selection of the most desirable alternative in light of the overall goals of the enterprise at the time the decision is made; and,

(vi) periodic re-evaluation and monitoring.

The following discussions will present a brief analysis of the process and an overview of the alternatives that are available.

\subsection{Identification}

Of the functions encompassed in risk management, the identification process is probably the most critical. A failure to recognize the existence of one or more potential events can result in financial disaster. It is usually costly and difficult to arrange financial protection against losses attributable to a particular event after it has occurred. Since risk management deals with potential financial costs of extreme magnitude, the importance of being able to anticipate the exposure before the loss occurs can hardly be overstated. It is unfortunate that there is no scientific method of approaching the identification process.

Several approaches to the identification process have been suggested. Most of these consist of a systematic evaluation of the financial and operational characteristics of the enterprise and its relationship to other enterprises and the environment. These approaches are of a check list variety, requiring the manager to investigate virtually every aspect of the enterprise, including its contracts with suppliers and users, alternative sources of supply and for distribution, sources and uses of funds, financial capacity, the operations used by the enterprise in pursuit of its basic function, dependence on personnel, distribution and location of assets, and many other items. The objective of these suggested approaches is, of course, to guard against the possibility that potential causes and sources of disruption will be overlooked.

In spite of the fact that substantial effort has been devoted to devising a systematic identification procedure, success is still heavily dependent upon the imagination and intuition of the individual(s) responsible for the task. The most difficult part of the identification process is that it requires that the manager be clairvoyant. Some of the pure risk events for which plans must be made are events which the enterprise has never experienced. Even worse, socioeconomic and technological changes may result in new risks with which there is not only a lack of experience within the enterprise, but even among all other enterprises of a similar nature. The area in which these changes and problems have been the greatest is that of legal liabilities.

The law is seldom static. The standards of care by which enterprises are judged, the circumstances under which they may be held legally liable, the variety of victims to whom they can be held responsible, and the costs that may be imposed upon them are in a constant state of change. These changes have generally been of liberalizing 
variety from the point of view of the victim, i.e., they have held the offending enterprise responsible under an expanding set of circumstances. Therefore, adequately identifying exposures requires that the manager not only be familiar with the basic requirements of the law, but also be able to predict changes which are sometimes extremely difficult to anticipate. To manufacturers and suppliers of services, the explosion in product and professional liability lawsuits and the changing (more liberal) interpretations of occupational injuries and diseases are all too familiar examples of these problems.

It is possible to summarize the identification process as a type of sleuthing exercise, attempting to discover all those pure risk events which may adversely affect the enterprise.

\subsection{Evaluation of potential costs}

Following an identification of the pure risks that face the enterprise, the next step is to determine the costs that might be incurred if any of these events should take place. The reason for selecting this as the second phase, rather than turning to some other function, is that the objective should be to eliminate risks from further consideration as quickly as possible. And the best way to accomplish this is to identify those events whose impact would be of little or no importance.

Two difficulties may be encountered in the evaluation process. First, it is necessary to recognize that two or more events may occur simultaneously. And while the financial impact of any one of these events alone may be unimportant, the simultaneous occurrence of many of the events may be disruptive. For example, the destruction of a warehouse by fire will not only involve the cost of the structure, it will also involve the inventory stored therein. Therefore, even though the destruction of either the warehouse or the inventory alone might not result in a financial burden, the actual loss will entail both items. This phenomenon must be explicitly recognized before the decision is made to eliminate individual events from further consideration.

The second difficulty encountered in the evaluation process is that the event may give rise to both direct and indirect costs. Legal liabilities are the best case in point. Claims against an enterprise alleging wrongdoing will entail not only the reparation paid to the victim, but will also include several other costs as well. On the one hand, the process by which settlement is reached will entail additional costs. Depending on the nature of the case, these costs may be minimal or they may be extremely large. Failure to recognize these costs will result in a significant underestimation of the total potential costs of legal liabilities.

In addition to settlement activities, there may be other kinds of indirect costs resulting from legal liabilities. For example, if the alleged wrongdoing involves faulty workmanship or a faulty product produced by the enterprise, the existence of the claim and subsequent notoriety may seriously affect the market position of the enterprise and therefore its continued existence. The necessity for product recall even further adds to the cost. As a result, the ultimate costs of legal liabilities may be substantially in excess of the payments to victims and the costs incurred in litigation and other settlement proceedings. 
Losses to property will also entail both direct and indirect costs. The most serious of the indirect type results from the fact that damage to property generally implies a loss of use as well as the costs necessary to restore or replace. Unless the property was unneeded by the enterprise, loss of use generally implies that the enterprise will either incur substantial additional costs for the use of substitute property, or an interruption in the activities of the enterprise, or both. This interruption can have serious financial consequences which will last for several periods. When significant property damage is involved, experience indicates that losses in revenues and additional costs resulting from loss of use can be significantly greater than the costs of replacement or restoration. Unfortunately, far too often enterprises ignore or overlook these costs in the evaluation of the exposures to property damage.

\subsection{Estimation of the frequency of occurrence}

Having identified the potential pure risk events and evaluated the possible financial impact, the third step involves estimating the frequency with which such events might occur. The major difficulty encountered in this process is simply a lack of information. What is needed, for each type of event, is an estimate of the possibility that the event will not occur, that it will occur once, that it will occur twice, and so forth, over the period for which plans are being made. From this information it would be possible to determine the most likely number of times that the event might be expected to occur and the possibility that it will occur with some frequency other than that expected.

As might be anticipated, there is no readily available source of information that might be used to provide these estimates for most enterprises. In part this is attributable to the fact that pure risk events keep changing. However, it is principally attributable to the fact that, for most of these events the data have simply never been gathered in a form that would be adaptable to the peculiar characteristics of each enterprise. There are some noteworthy exceptions to the general rule. For example, in some countries the public concern with occupationally-related injuries and diseases has given rise to government-organized efforts to gather and analyze data. These data are then available on the basis of industry, occupation, and size of the enterprise.

The importance of an estimate of the frequency of occurrence can only be appreciated when it is recognized that an appropriate evaluation of alternatives is critically dependent upon it. As a result, those enterprises that are sufficiently sophisticated in risk and insurance management establish an elaborate procedure for recording their own experience. This is extremely useful if the enterprise is sufficiently large so that the results are meaningful. If the enterprise is not sufficiently large, then it may enter into agreements with other similar enterprises providing for a mutual recording and exchange of information. Before such information can be generated, the enterprise faces substantial uncertainty.

One of the difficulties in estimating the frequency of events was suggested in the discussion regarding the evaluation of potential costs. Different pure risk events may occur simultaneously. In fact, such events may occur automatically in conjunction with others. This possibility must not be overlooked. 
From the estimates of frequency of events and estimates of the potential costs that would result if the events were to occur, it is possible to derive several pieces of information. For example, it is possible to estimate the probability that retained losses will reach particular levels under various self insurance options and to evaluate the premium being charged for different insurance programs.

\subsection{Alternatives}

Generally speaking, there are four alternative solutions that may be employed in risk and insurance management. These solutions include : (i) deterrence; (ii) transfer (generally by contract) to some entity other than through the purchase of insurance ; (iii) assumption of the risk (typically referred to as self insurance); and, (iv) the purchase of insurance from a commercial insurance company.

The purpose of the following discussion is to explain the basic nature of these activities and to indicate the circumstances in which the alternative is most appropriate.

Deterrence. There are several circumstances in which programs may be devised for reducing the enterprise's exposure to pure risk events. These programs are typically referred to as loss prevention programs (which is somewhat of a misnomer). They may be useful for reducing the frequency with which events occur, for reducing the financial impact of the event on the enterprise, or for both. They may range from the most simplistic changes in the operating or physical characteristics of the enterprise to highly sophisticated programs designed to avoid such problems as faulty workmanship, defective products, and so forth. And, they may be programs which entail substantial capital expenditures or they may be programs which are very short run in nature, easily modified, with no long term commitment. As an overall matter, there are a plethora of potential loss prevention programs with widely differing characteristics.

Loss prevention programs have been developed for virtually every one of the major types of pure risk events faced by enterprises including occupational disability of employees, damage or conversion of property, legal liabilities and nonperformance by independent contractors and employees. In the case of occupational disabilities, there are a wide variety of available approaches. Some of these approaches are principally geared to educating employees regarding their exposure to disability in their occupation. Periodic seminars, indoctrination programs for new employees, and notification of dangerous conditions are procedures which are considered by all but the most unsophisticated enterprises.

Other programs concerned with occupational disability represent attempts to reduce the hazards to which employees are exposed. These include such activities as installing safety devices on equipment, removing or isolating toxic material, or even modifying the process by which the goods or services are created by the enterprise. Finally, many enterprises have established contingency plans for providing prompt medical treatment for injured employees and for providing medical and vocational rehabilitation, thereby attempting to minimize the impact of the injuries that do occur.

In the case of damage to or conversion of property, programs may include such activities as the general maintenance of equipment and facilities, security programs, 
installation of fire detection and fire fighting equipment, and training employees in fire fighting techniques. Programs on a larger scale include choice of construction materials and even the selection of a site for the facilities. The latter is frequently important for not only avoiding obvious problems such as floods and earthquakes, but also to guarantee the ready availability of fire fighting facilities.

Loss prevention programs for the exposure to legal liabilities are typically more complicated than for either of the other two major categories of pure risk events. They are as varied in purpose as the potential areas of legal liabilities are different. For enterprises in which the general public is frequently on the premises, consideration must be given to maintaining the premises in a manner that is safe and secure. For enterprises that produce consumer products, special programs are available that are designed to detect defects, communicate adequate warning regarding the use of the product, test the product for unforeseen hazards, facilitate recall of the product if it is determined to be hazardous after it has been distributed, and so forth.

Of course, in many countries, the decision about whether to implement a loss prevention program is frequently removed from the discretion of management. This is due to the fact that the benefits that are realized when loss prevention programs are implemented extend beyond the enterprise itself. They tend to benefit the whole of society. Therefore, it is felt that the decision should not be left to the vagaries of any individual or small group of individuals, who may not recognize the full benefits of the programs.

The imposition of loss prevention programs is most widespread in the areas of occupational disability and legal liability. The two most prominent requirements of this variety in the United States are embodied in the Occupational Safety and Health Act of 1970 (OSHA) and the Consumer Product Safety Act of 1972. The purpose of OSHA is to provide employees with wholesome and safe places to work. It stipulates procedures that are to be followed by employers, and imposes a system of fines and penalties for violations. Among all industries, OSHA may have had its greatest impact on the occupational disability hazards of construction.

There are a large number of sources of assistance to enterprise in the identification, design, implementation, and even management of loss prevention programs. Since the programs are designed to reduce the cost of pure risk events, insurance companies have traditionally provided loss prevention assistance as an ancillary service to the insurance agreement. In fact, insurance companies will frequently require that certain loss prevention programs be undertaken as a condition to their willingness to provide insurance ; and, they will often grant concessions in the premium charge when optional loss prevention programs are undertaken. In addition to insurance companies and their affiliates, loss prevention services can be obtained from several other enterprises. The more sophisticated insurance brokers have the capacity to provide loss prevention assistance; and, there are a few entities that are not connected with the insurance industry that provide these services.

The major difficulty in the loss prevention area is not the identification of alternatives or the implementation of the program, but rather it is in the evaluation of their desirability. On a theoretical basis, it is easy to specify when a loss prevention 
program should be undertaken : it should be undertaken whenever the costs associated with its design, implementation and maintenance are more than offset by the savings to the enterprise resulting therefrom.

The problem arises when attempts are made to measure the potential costs and benefits of different loss prevention programs. With regard to the benefits, it is frequently difficult to devise more than an extremely crude estimate of the impact that the program will have on the frequency and severity of losses. Further, there are indirect benefits whose value is subject to little more than mere speculation. For example, a reduction in employee injuries and the installation of safety programs are alleged to increase morale and efficiency. However, only imprecise estimates may be derived for these benefits. The only concrete measure of benefits is the premium reduction that is offered by the insurance company (and, if applicable, the reduction in fines and penalties). Similar problems are encountered in attempts to measure the costs.

In spite of these difficulties, frequently the net benefits of such programs are intuitively obvious. And, among all other alternative solutions to the pure risk problem, loss prevention is the most widely accepted.

Transfer Other Than Through Insurance. There are a number of opportunities available to enterprises to shift the burden of pure risk events, in whole or in part, to some other entity. In fact, such transfers are possible whenever the enterprise enters into a contractual arrangement with outsiders for either the sale or purchase of goods or services. For example, if the enterprise enters into an arrangement to lease equipment or facilities for its own use, the contract may stipulate that the provider (lessor) bear the burden of any damage to, or destruction of the property. The transfer may include responsibility for losses regardless of cause, or it may be limited. For example, it might specify that the damage will be the lessor's responsibility unless it is caused by the negligence of the enterprise or its employees, in which event the enterprise shall be responsible for the costs.

Contractual transfers or sharing of responsibility may include the liability risk as well as the risk of physical damage to the property owned or used by parties to the contract. As in the case of transfers regarding damage to, or destruction of property, the transfer may be conditioned upon the circumstances giving rise to the liability. Using the leasing example, the contract may provide that the lessor be responsible for all legal liabilities arising out of the use of the equipment or facilities, or it may limit the responsibility of the lessor to those liabilities which are attributable, in whole or in part, to the actions of the lessor and his employees.

Contractual agreements of this variety are extremely complicated, and easily subject to disputes regarding interpretation. Their discovery and evaluation is one of the most difficult aspects of the risk identification and evaluation process.

Two questions need to be specifically addressed. One of these is : why would an enterprise be willing to assume additional obligations for pure risk events ? The traditional answer to this question is that the party is at a competitive disadvantage, and therefore is being forced to assume such responsibilities in order to engage in the 
activity that he desires. This answer is misleading and tends to obscure basic considerations.

It is quite possible that between the parties to a contract, one is distinctly better able to bear the risk in a relatively costless and expeditious fashion. For example, between a buyer and seller of goods, the seller may be much better able to prevent damage to the goods in transit (because of his greater knowledge of the hazards or his established relationship with shippers). Since the buyer has less capacity to deal with the problems, or less expertise, he would have to expend greater effort (and hence endure greater costs) to provide the same protection. As long as the additional consideration the buyer is willing to provide for this service is greater than the costs incurred by the seller, the arrangement is mutually advantageous. It is not a matter of the seller being victimized because of a competitive disadvantage.

This leads directly to the second question : how is the desirability of a contractual transfer to be evaluated? From the point of view of the transferor, as long as the consideration that must be given the other party for assuming the risk is less than the costs that would be incurred if the risk were retained, the transfer is desirable. Attention must be given to the costs of loss prevention and the costs of risk bearing (including among other considerations, the premiums for any insurances that may be involved). A rule that is frequently applied is that responsibility should be placed on the party who has the greatest control over the occurrence of the event. But there are several exceptions to this rule, and it should not be blindly adopted in all situations.

Self Insurance. As a generic concept, self insurance exists whenever the costs attributable to pure risk events are directly absorbed by the financial resources of the enterprise. Although the risk management programs of even the largest enterprises incorporate at least some use of insurance, the tendency over the past decade has been to substantially reduce reliance on the insurance industry and to assume internally a greater share of the responsibility for losses and related activities. The tendency toward self insurance and the proliferation of alternative programs is attributable to a variety of considerations including, among others :

(i) the reluctance of insurance companies to provide protection for certain types of losses (e.g., product liability) ;

(ii) inflationary pressures which alone would increase at least the nominal level of self insurance ;

(iii) a clear recognition of the fact that insurance premiums contain margins for profit, marketing and administrative activities, some of which are of no real benefit to the individual policyholder ;

(iv) a belief on the part of some enterprises that the pricing practices followed by insurance companies tend to result in premiums that are excessive for their particular exposures to loss ;

(v) cash flow considerations arising from substantial time lags between the premium payment under traditional insurance programs and the payment of losses and related expenses ;

(vi) the increased willingness, albeit with some reluctance, of insurance companies to offer alternative financing arrangements which entail greater internal responsibility 
on the part of the policyholder while still providing protection against the financially disruptive occurrences; and,

(vii) the availability of services needed to successfully operate a self insurance program.

One comment may be in order. The reluctance of commercial insurance companies to provide certain types of protection is well documented. This is sometimes attributable to a lack of confidence in their ability to accurately predict the losses that will arise under the insurance. As a result, premiums may incorporate a substantial margin for error. The basic question from the point of view of the risk manager is whether the insurance industry has overreacted, charging prices that are based on an unnecessarily pessimistic assessment of future conditions.

There are a wide variety of ways in which self insurance programs can be structured and funded. Although the traditional approach has concentrated on the use of stipulated deductibles applied to each loss (generally referred to as the "per occurrence deductible"), there are numerous other possibilities. The enterprise could co-insure each loss by retaining a stipulated fraction, intentionally purchase inadequate coverage for the potential loss, or purchase insurance for losses up to a certain level, self insure the next "layer", and purchase insurance for losses above that layer. Of course, it is possible to employ two or more of these options for even a single exposure.

Another alternative structure is to concentrate on the total losses arising in a particular time frame. Under this approach the enterprise could retain responsibility for each loss, regardless of size, occurring in a particular time frame until the total value of the retained losses reaches a stipulated amount (typically referred to as the "aggregate deductible"). Once the threshold is reached, all subsequent losses in the time frame would be the responsibility of the insurer. In fact, this approach could be used in conjunction with a per occurrence retention program to protect the enterprise against the possibility that an unusually high frequency of losses gives rise to a sum of deductibles (or other types of per occurrence retentions) which imposes greater retained losses than is desired.

In short, the number of alternative structures is limited only by the imagination of the risk manager and the flexibility of underwriters.

There are basically three methods of financing self insurance programs. These include : (i) an unfunded program with ensuing costs being taken from cash flow; (ii) an internal funded program with costs being taken from working capital or earned reserves; or (iii) an external funded program using a trust or other mechanism. The choice among these alternatives is influenced by a variety of factors.

(i). Unfunded Self Insurance Programs. The principal advantage of an unfunded self insurance program is its simplicity. However, the usefulness of an unfunded approach is limited. It is most appropriate when the costs arising from the pure risk events are relatively stable. And, it may be appropriate if it is easily and relatively costless for the enterprise to obtain funds to cover the losses when they do arise. For example, if the enterprise has ready access to large amounts of funds at little or no cost, then it can simply borrow the money it needs to cover the costs of the pure risk events as they arise and repay the borrowings out of future income. 
As a practical matter, unfunded self insurance programs with significant exposures are possible only for the largest enterprises. However, it is also clear that virtually all enterprises elect to engage in some unfunded self insurance activity.

(ii). Internal Funded Self Insurance Programs. When the costs of pure risk events are not sufficiently predictable, or stable, to absorb them out of cash flow and when funds would be difficult to obtain after a loss, an alternative is to set aside funds which would be available for use when the event does occur. The funds may be set aside in the form of higher working capital or an earmarked reserve. While it is not necessary to keep such funds in the form of cash, it is necessary that the investments for such funds be liquid and that their value be secure. If this is not the case, then the funds may not be available when they are needed. The fact that the investments must be both liquid and secure implies that the enterprise will incur some opportunity cost by virtue of a funded program. This opportunity cost will be due to the fact that the enterprise will not be able to use the funds in the furtherance of its own activities. Therefore, the earnings on funds maintained for a self insurance program will probably be less than they would be if the enterprise had discretionary use of them.

(iii). External Funded Programs. Among the alternatives, captive insurance companies have been the most popular external mechanism for funded self insurance programs dealing with property and casualty exposures. Such companies are capitalized by the enterprise and payments are periodically made to the captive in the form of insurance premiums. The captive assumes responsibility for the pure risk events of the parent and then purchases protection from reinsurance companies. The relationship between the captive and its parent enterprise may be more or less formalized. At one extreme, the captive may behave just as if it were a commercial insurance company, providing claims, loss prevention, and other ancillary services, for which charges are made to the parent on an arms length basis. These services may be provided either by employees of the captive or they may be purchased from insurance companies or other servicing entities.

Students of the captive insurance company phenomenon have proposed several possible advantages to the use of such companies. However, for the most part these advantages are due to institutional or governmental restrictions existing in many countries. For example, there are definite advantages to being able to purchase insurance through the reinsurance arrangement as opposed to purchasing insurance in the open market using normal channels. In the reinsurance market, insurance companies have much greater flexibility and are much more able to create protection that is tailored to the individual needs of the enterprise.

Since most of the advantages of captive insurance companies are attributable to regulation by governments, in countries where such regulation does not apply these advantages will not be present. However, there is a potential use of a captive company which could be advantageous to the parent and which is generally not possible under an internal funded self insurance program.

In addition to the risks of the parent, the captive could insure the risks of other unrelated enterprises or could reinsure other insurance companies. This could give rise to several advantages for the parent. First, any earnings resulting from such trans- 
actions would, of course, become earnings of the parent. This will lead to a reduction in the net cost of protection to the parent. Second, to the extent that the captive's employees gain greater experience with a variety of insurance problems, such experience will be available in solving the risk and insurance management problems of the parent. Finally, and perhaps most important, diversification of the captive's insurance activities should give it greater financial stability and capacity. It must be remembered that the actual protection afforded the enterprise by its captive insurance company is no greater than the financial stability and capacity of the captive. If the captive is financially unstable, the costs will simply fall back on the shoulders of the parent enterprise.

Commercial Insurance. Responding to the increasing propensity to self insure and competitive pressures, insurance companies have developed a variety of alternative programs designed to more accurately reflect the actual exposure of the individual enterprise and its use of ancillary services. In fact, it is now possible to purchase ancillary services (such as safety engineering and claims investigation and settlement) on a fee-for-service basis without purchasing insurance, in what has become widely referred to as "unbundled" programs.

Alternative procedures for more accurately pricing insurance protection to reflect the actual claims or loss propensity of the enterprise are numerous. Starting with procedures that base future premiums on actual loss experience, companies have extended the approach to the point where premiums for the current policy period are modified to reflect the actual losses incurred during this period. In its pure form, the latter arrangement results in a program in which there is no actual insurance protection in the traditional sense and the insurance company is merely providing ancillary services. If the timing and amount of premium payments more follow the actual insurance company expenditure pattern regarding payments of losses and the timing of ancillary services, the enterprise will avoid the loss of use of funds that characterizes traditional insurance arrangements. Such plans are generically referred to as cash flow programs.

To accommodate the desire to avoid wide fluctuations in premium payments from one period to the next, programs that link premiums to actual loss experience may be modified to limit such fluctuations to levels that the enterprise desires. These approaches simply reintroduce insurance in the financing arrangement.

In the extreme, arrangements with insurance companies may result in programs that are analogous to self insurance programs using an external trust whereby the insurance company acts as the trustee. If the enterprise has discretion to insure the financial stability of the trust and arrange for ancillary services with providers other than the trustee, it will have essentially created a captive insurance company with similar access to the reinsurance market.

The plethora of approaches offered by different insurance companies increase the difficulty of making comparisons among competing programs. In addition to analyzing the financial stability, specific coverages, and the capability of different insurance companies to provide services, it is frequently necessary to compare pricing formulas and fee-for-service arrangements that differ in subtle respects. This situation has dramatically increased the role and importance of the broker. 


\subsection{Choosing among alternatives}

Although administrative costs, premium payments, and other known charges under different programs may vary widely in both timing and amount, as previously indicated the most difficult problem involves choices between the risk and anticipated savings. In many cases, the alternative which offers the possibility for the greatest savings also entails the greatest risk that these savings will not only fail to materialize, but that the enterprise will suffer serious financial disruption. Therefore, it is extremely important to identify and evaluate the risk under each strategy.

Analyses by social scientists and others have indicated that individuals have substantially different attitudes toward risk. Some individuals tend to be unaffected by the presence of risk, others tend to be attracted to it, and others tend to avoid it. One of the most difficult problems of the risk manager is identifying the preferences of owners or shareholders with regard to the risk and return relationship. There is no mathematical formula, with solely objective components based on financial or other readily measurable characteristics, that can be uniformly applied across all enterprises to yield the optimal solution.

One other aspect of the selection process follows from previous discussions. Since the risk resulting from any alternative solution is an integral part of the total risk faced by the enterprise, it is not appropriate to devise a solution to the risk management problem in isolation. Choosing a particular strategy without taking cognizance of the other risks faced by the enterprise would be similar to selecting a particular security without assessing its impact on the overall volatility of the investment portfolio. Furthermore, the decision to adopt a particular self insurance strategy in anticipation of some net savings must be made in light of the risk and return relationship existing for other alternatives that the enterprise has available to it.

In actual practice, the integration of risk management decisions with the overall goals and position of the enterprise is generally provided by rules of thumb indicating that the program should limit the possibility of uninsured losses during a particular time horizon to some stipulated fraction of revenues, working capital, earnings per share, or other financial measure. Evaluating alternatives to determine whether they conform to these restrictions is not a simple exercise.

\section{Conclusion}

The choice among alternatives in the risk and insurance management problem is a decision that must be based on judgment and the personality of the enterprise. Mathematical decision rules can be helpful and theories of probability and statistics can be useful for better defining the differences among the alternatives. Financial analysis techniques can also be useful for better identifying the alternative costs. Nevertheless, the fact that there is hardly ever sufficient, reliable information regarding many of the variables that must be considered or the basic nature of pure risk events results in a situation in which there is substantial room for differences of opinion among knowledgable practitioners. 